\title{
Schwannoma del plexo braquial: reporte de un caso y revisión de la literatura
}

\author{
Guillermo Moreno Flores, ${ }^{*}$ Luis Israel Llerena Bejar, ${ }^{*}$ Eva Ruvalcaba Limón, ${ }^{\ddagger}$ \\ Julio Ramírez Bollas, ${ }^{\ddagger}$ Carlos Fernando Gómez Cuevas, ${ }^{\ddagger}$ Jorge Alberto Salazar Andrade, ${ }^{\ddagger}$ \\ Betsabé Hernández Hernández, ${ }^{\S}$ Felipe Villegas Carlos," Juan Alberto Tenorio Torres, \\ Carlos Alberto Domínguez Reyes, ${ }^{* *}$ Verónica Bautista Piña, ${ }^{\ddagger}$ Javier Hernández Hernández, ${ }^{\ddagger}$ \\ Rafael Topete Estrada§§
}

\section{RESUMEN}

El schwannoma es el tumor más común de los nervios periféricos, la transformación maligna es extremadamente rara, los tumores están compuestos por células de Schwann, que sostienen las fibras nerviosas periféricas y son de origen neuroectodérmico. Objetivo: Presentación del caso clínico de una paciente con schwannoma del plexo braquial. Material y métodos: Se revisó el expediente clínico en el Instituto de Enfermedades de la Mama, FUCAM, AC Presentación del caso: Mujer de 77 años con autodetección de nódulo axilar derecho palpable, con mastografía y ultrasonido no FUCAM el 30/10/2018 BIRADS 4a. Acude al instituto por tumoración axilar, se solicitan estudios de imagen que reportan BIRADS 2 , por lo que se realiza biopsia guiada por ultrasonido, con reporte de neoplasia fusocelular, se realiza revisión de laminillas por dos patólogos y se decide en sesión conjunta biopsia escisional de nódulo axilar derecho. Con reporte patológico del 04/04/2019: schwannoma de $5 \times 3$ centímetros, totalmente resecado. Conclusiones: Los tumores del plexo braquial son poco frecuentes, de inicio no podemos descartar tumor primario de mama, o alguna metástasis. Knight reportó serie de 234 schwannomas entre 1984 y 2004, de los cuales 94 (40\%) surgieron del plexo braquial, se usó la resonancia magnética como método de imagen de primera elección; sin embargo, el ultrasonido puede ser suficiente siempre y cuando se acompañe de una mastografía, deberá confirmarse por patología para descartar lesión maligna. Todos los autores sugieren que el tratamiento adecuado es la extirpación y preservar la función nerviosa y vascular. No hay que olvidar que ante una tumoración axilar, siempre debe descartarse etiología maligna.

\begin{abstract}
Schwannoma is the most common tumor of the peripheral nerves, the malignant transformation is extremely rare, the tumors are composed of Schwann cells, which support the peripheral nerve fibers and are of neuroectodermal origin. Objective: Presentation of the clinical case of a patient with schwannoma of the brachial plexus. Material and methods: The clinical record was reviewed at the Institute of Breast Diseases, FUCAM, A.C. Case presentation: 77-year-old female, with palpable right axillary nodule self-detection, with mammography and non-FUCAM ultrasound on 10.30.2018 BIRADS $4 a$. He comes to the institute for axillary tumor, imaging studies are requested that report BIRADS 2, so an ultrasound-guided biopsy is performed, with a report of spindle cell neoplasia, a revision of the lamellae is carried out by two pathologists and a joint session is taken of excisional biopsy of right axillary nodule. With pathological report of 04.04.2019: schwannoma of $5 \times 3$ centimeters, totally resected. Conclusions: Brachial plexus tumors are infrequent, we cannot rule out primary breast tumor or any metastasis at the beginning. Knight reported series of 234 schwannomas between 1984 and 2004, of which 94 (40\%) arose from the brachial plexus, used MRI as the imaging method of first choice, however ultrasound may suffice as long as it is accompanied by a mammogram should be confirmed by pathology to rule out malignant injury. All authors suggest that proper treatment is excision and preserving nerve and vascular function. It should not be forgotten that in the event of an axillary tumor, a malignant etiology must always be ruled out.
\end{abstract}

* Residente del Curso de Alta Especialidad en Diagnóstico y Tratamiento de los Tumores Mamarios.

¥ Médico adscrito.

$\S$ Coordinadora de consulta externa.

"I Director Médico.
ฯ Director de Enseñanza e Investigación.

** Director General Médico.

㧊 Jefa de Anatomía Patológica del Instituto de Enfermedades de la Mama, FUCAM, A.C.

$\S \S$ Asesor de redacción, Vicepresidente de AMEGM. 
Palabras clave: Schwannoma, tumor de plexo braquial, tumoración axilar, patrón de Antoni.

\section{INTRODUCCIÓN}

El schwannoma es el tumor más común de los nervios periféricos, la transformación maligna es extremadamente rara, los tumores están compuestos por células de Schwann que sostienen las fibras nerviosas periféricas y son de origen neuroectodérmico. ${ }^{1}$

Suelen ser lesiones solitarias con aspecto de nódulos firmes, elásticos, bien delimitados y encapsulados. $^{2}$

Histológicamente, son tumores con alta celularidad, incluso puede existir atipia en algunos de ellos. Se manifiestan clínicamente corno masas palpables que originan dolor radicular y déficits neurológicos tales como parestesias, debilidad y atrofia muscular en el territorio nervioso involucrado. ${ }^{2}$ La resonancia magnética es el método de elección, el ultrasonido permite sospecharlo; ${ }^{3}$ sin embargo, puede existir un diagnóstico erróneo y pensar en patología maligna, de ahí la importancia de un abordaje adecuado.

\section{Presentación del caso}

Mujer de 77 años con autodetección de nódulo axilar derecho palpable, con mastografía y ultrasonido no FUCAM el 30/10/2018 con BIRADS 4a. A la exploración inicial en el instituto, se encuentra mama izquierda sin nódulos palpables, mama derecha sin nódulos palpables, axila derecha con ganglio apical móvil de $3 \mathrm{~cm}$, por lo que se solicita ultrasonido mamario y axilar el 20/11/2018, dando como resultado tejido fibroglandular con patrón homogéneo graso, sin alteración focal. Piel, complejos areola pezón, grasa subcutánea y espacios retromamarios normales en identificar hilio graso ni cortical, mide $4.8 \times 1.8 \times 3$ centímetros, es palpable y se ubica adyacente a la arteria y vena axilar derecha (la vena resulta con compresión significativa y reducción del flujo de retorno), es probable que se ubique intramuscular. No hay evidencia de adenopatías en nivel I de axila derecha, categorizado como BIRADS 2 (Figura 1).

Por lo anterior, se realiza biopsia guiada por ultrasonido, con Folio Q18-4035 que reporta neoplasia fu-
Keywords: Schwannoma, brachial plexus tumor, axillary tumor, Antoni pattern.

socelular; debido al reporte, se solicita nueva revisión de laminillas por un segundo patólogo y se envía a sesión conjunta multidisciplinaria, donde se programa para biopsia escisional de nódulo axilar derecho, que se realiza el 14/01/2019. Con reporte patológico definitivo Q19-1181 del 04/04/2019, biopsia escional de axila derecha: schwannoma de $5 \times 3$ centímetros.

Posterior a cirugía, se realiza sesión de morbilidad el 24/04/2019, donde se concluye candidata a vigilancia únicamente, no necesita tratamiento adyuvante por no tratarse de una neoplasia maligna.

\section{Discusıón}

El schwannoma es un tumor benigno de la vaina del nervio, los tumores primarios del plexo braquial son
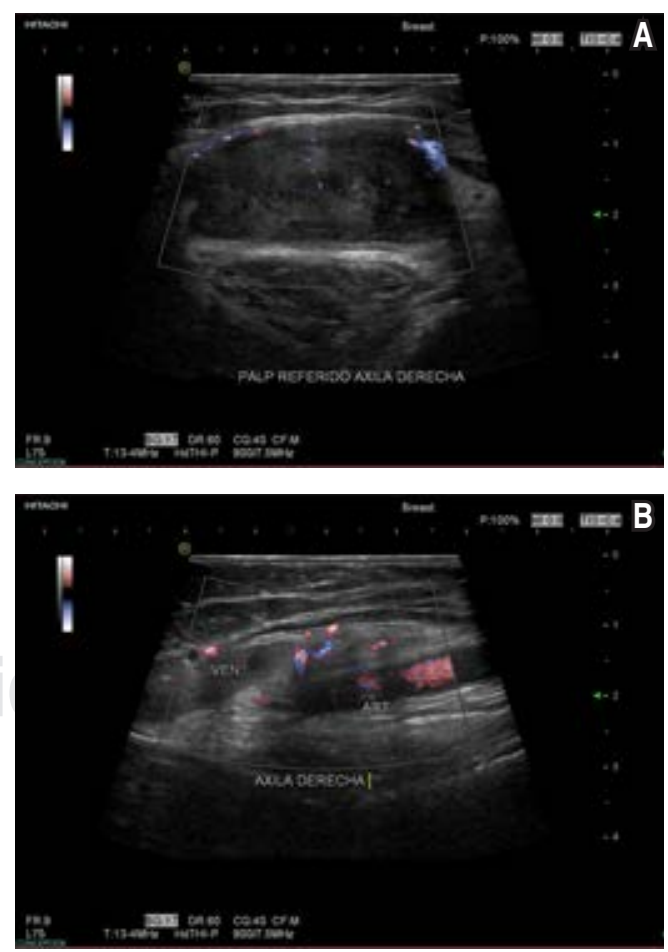

Figura 1: A) Relación anatómica de la axila y el tumor. B) Tumoración axilar. 
Figura 2:

Schwannoma. A) Áreas densas fusocelulares formando empalizadas (patrón Antoni A). B) Áreas más laxas (patrón Antoni B).

C) Inmunorreactividad para S-100 con expresión difusa.
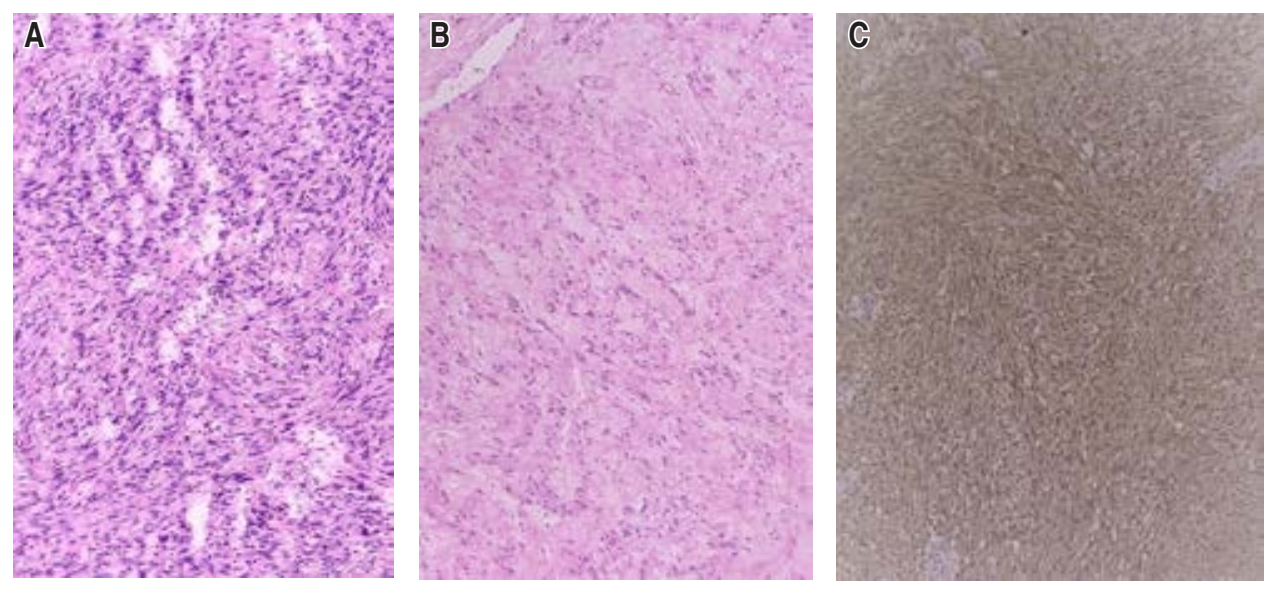

raros y se pueden dividir en dos grupos: los tumores periféricos de la vaina neural y los tumores periféricos de la vaina no neural. Los schwannomas, junto con los neurofibromas, son tumores periféricos de la vaina nerviosa y rara vez afectan el plexo braquial. Los schwannomas surgen esporádicamente y son una característica principal de dos enfermedades tumorales hereditarias, la neurofibromatosis tipo $2 \mathrm{y}$ la schwannomatosis, por lo cual representan un reto para el cirujano.

Desde el punto de vista patológico, los schwannomas son neoplasias benignas originadas de los nervios periféricos que histológicamente se caracterizan por la proliferación de células de Schawnn neoplásicas que tienen dos patrones de crecimiento. A) El primero conocido como patrón de Antoni A donde se observan células fusiformes con un núcleo elongado y alargado, un amplio citoplasma eosinófilo dispuesto en fascículos celulares entrecruzados formando empalizadas con los núcleos y cuerpos de Verocay que corresponden a la zona sin núcleos, ubicados entre las empalizadas. B) El segundo patrón de Antoni B caracterizado por áreas hipocelulares en el que las células fusiformes están separadas por matriz extracelular mixoide rica en mucopolisacaridos. C) Estas células neoplásicas se caracterizan por la inmunorreactividad uniforme para S-100 (Figura 2). ${ }^{4}$

Los tumores del plexo braquial son poco frecuentes, inicialmente no podemos descartar tumor primario de mama e incluso de metástasis. Knight ${ }^{1}$ tiene la serie más grande con 234 schwannomas entre 1984 y 2004, de los cuales 94 (40\%) surgieron del plexo braquial, usó la resonancia magnética como método de imagen; sin embargo, el ultrasonido puede ser suficiente siempre y cuando se acompañe de una mastografía, deberá corroborarse por histopatología y así poder excluir tumoración maligna.

Clínicamente, se presenta como una tumoración palpable (60\%), parestesias (44\%) y dolor irradiado (44\%) como lo describe Roldán. ${ }^{5}$ Sin embargo, puede tener presentaciones atípicas como elevación de la escápula, escalenos y dolor en pectoral mayor y menor, nuestra paciente no tenía ninguno de estos síntomas, que puede ser debido al tamaño inicial de 3 centímetros, un mayor tamaño implica mayor compromiso neurovascular.

Los schwannomas rara vez afectan el plexo braquial, representan aproximadamente el 5\%, y son incluso más raros en región supraclavicular; sin embargo, existe un caso reportado en 2018 por Vučemilo ${ }^{3}$ en una paciente de 49 años. Los autores mencionan que se requiere precaución adicional en los procedimientos de aspiración con aguja fina o biopsia con aguja gruesa debido a la posible lesión iatrogénica del nervio y las estructuras adyacentes. ${ }^{3}$

El diagnóstico diferencial es amplio, incluye la presencia de adenopatías, lipomas, angiomas, neurofibromas, parangangliomas, linfoma, fibrosarcoma, leiomiosarcoma, sarcoidosis, tuberculosis, tumores primarios o metastásicos y síndrome de hombro doloroso, por lo que es muy importante considerarlos como parte del abordaje diagnóstico. ${ }^{5}$ 
Los schwannomas envuelven de manera total o parcial a la vaina del nervio y estructuras cercanas, por lo tanto las principales secuelas son el déficit neurológico, así como lesiones vasculares en menor frecuencia, que incluso pueden provocarse en la toma de biopsia con aguja gruesa.

\section{Conclusiones}

Algunos autores ya mencionados refieren que la indicación para el tratamiento quirúrgico es la existencia de una disfunción neurológica, de dolor y/o crecimiento rápido; sin embargo, ante una tumoración en región axilar, siempre se debe considerar la cirugía para establecer correctamente el diagnóstico histopatológico, con el objetivo de descartar un tumor maligno debido a la alta incidencia de cáncer de mama asociado al diagnóstico tardío en países en vías de desarrollo.

Es importante recordar que la disección e identificación quirúrgica de las estructuras adyacentes es fundamental para tratar de preservar la función nerviosa tanto sensitiva como motora y/o evitar lesión vascular. En la medida de lo posible realizar biopsias guiadas por personal experimentado.

\section{Bibliografía}

1. Knight DM, Birch R, Pringle J. Benign solitary schwannomas: a review of 234 cases. J Bone Joint Surg Br. 2007; 89 (3): 382-387. doi: 10.1302/0301-620X.89B3.18123.

2. Rodríguez-Boto G, Moreno-Gutiérrez A, Gutiérrez-González R, Villar-Martín A, Arrez-Aybar LA, Serrano Hernando J. Neurinoma del plexo braquial simulando metastasis de adenocarcinoma de mama. Medicina (B. Aires); 2011; 71 (5): 459-461.

3. Vučemilo L, Lajtman Z, Mihalj J, Plašćak J, Mahović Lakušić D, Mužinić D. Brachial plexus schwannoma - case report and literature review. Acta Clin Croat. 2018; 57 (2): 366-371. doi: 10.20471/acc.2018.57.02.19.

4. Cortesía del Departamento de Anatomía Patológica del Instituto de Enfermedades de la mama FUCAM, A.C.

5. Roldán-Aviña JP, Herrera-Gutiérrez L, Palacios-García E, Romero-Vargas ME, del Álamo-Juzgado C, MuñozPozo F, de Valle Paredes-García M. Schwannoma del plexo braquial simulando adenopatía axilar. Revista de Senología y Patología Mamaria, 2015; 28 (4): 193-194. doi: 10.1016/j. senol.2015.07.001.

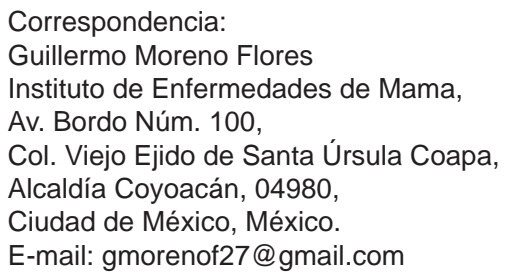

